\section{Programa de detección de neoplasias colorrectales en población mayor de 50 años}

FRANCISCO LÓPEZ-KÖSTNER ${ }^{1}$, UDO KRONBERG ${ }^{1}$, ALEJANDRO J. ZÁRATE ${ }^{1}$, ANA MARÍA WIELANDT ${ }^{1}$, ELIANA PINTO ${ }^{1}$, CRISTOBAL SUAZO ${ }^{1}$, PAULINA ORELLANA ${ }^{1}$, RODOLFO AVENDAÑO르, GUSTAVO BRESKY ${ }^{3}$, MIRKO CASTILLO ${ }^{4}$, JAIME LUBASCHER ${ }^{5}$, STANKO KARELOVIC ${ }^{6,7}$, MARCELO ROSS ${ }^{6,7}$, MISAEL OCARES ${ }^{8}$, FERNANDO RIQUELME $^{9,10}$, LUIS CONTRERAS ${ }^{11}$, BELISARIO VARGAS ${ }^{12}$, MIGUEL CORTÉS ${ }^{12}$

\section{A screening program for colorectal cancer in Chilean subjects aged fifty years or more}

Background: Mortality from colorectal cancer (CCR) in Chile has nearly doubled over the past 15 years. International studies have shown that CCR screening programs based on fecal occult blood test (FOBT) reduce CCR mortality. Aim: To analyze the results from a CCR screening model in people over 50 years. Material and Methods: Between 2007 and 2009, a prospective multicenter study was performed in seven major Chilean cities. FOBT using an immunological method, was measured in asymptomatic subjects aged 50 years or more, without risk factors. In patients with a positive FOBT, with symptoms or with family risk factors, a colonoscopy was indicated. Results: A total of 6348 subjects were assessed, FOBT was performed in 4938 of them, with a compliance of $77 \%$. The result was positive in $9.6 \%$. A total of 2359 colonoscopies were ordered, with an overall compliance of $50.1 \%$. Of the 1184 colonoscopies performed, adenomas and high risk adenomas were found in 304 (26\%) and $75(6 \%)$ patients, respectively. Thirteen patients were diagnosed with stage I and II CCR. Three of these lesions were excised endoscopically and 10 surgically. The detection rate of polyps, high risk adenomas and cancer was 75, 12 and 2 per 1000 screened individuals, respectively. Conclusions: This program allowed the early detection of an important number of high risk colon lesions, and all patients with CCR were diagnosed at early stages.

(Rev Med Chile 2012; 140: 281-286).

Key words: Adenomatous polyps; Colonoscopy; Colorectal neoplasms.

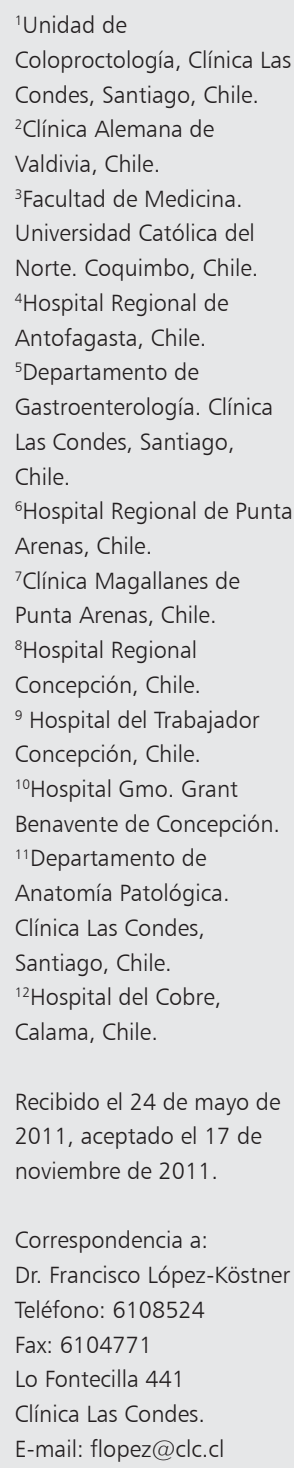

L a mortalidad por cáncer de colon y recto (CCR) se ha duplicado en el período 1983$12008^{1}$, constituyendo una de las causas de muerte más importantes por cáncer en nuestro país. En países desarrollados, el CCR se encuentra entre las primeras causas de muerte por neoplasias malignas ${ }^{2}$ y probablemente los factores ambientales sean los principales gatillantes de este tipo de tumores.

El CCR presenta algunas características que lo hacen particularmente susceptible de ser diagnosticado en etapas iniciales o mejor aun, ser susceptible de prevención secundaria ${ }^{3,4}$. La gran mayoría de los CCR presenta un desarrollo progresivo desde la etapa de pólipo adenomatoso, el cual luego de un período variable entre 6 y 10 años se podría transformar en cáncer (secuencia adenoma-carcinoma) $)^{5}$. Lamentablemente la inmensa mayoría de los pólipos no dan síntomas por lo que la única forma de interrumpir la secuencia 
adenoma-carcinoma es a través de programas de pesquisas. El CCR sintomático se diagnostica entre la $6^{\text {ta }}$ y $7^{\text {a }}$ década de la vida, por lo que la mayoría de los programas de pesquisa se inician desde los 50 años. Los exámenes utilizados en programas de pesquisas poblacionales deben poseer algunas características, como un bajo costo, buena sensibilidad y especificidad, ser fácil de aplicar, no invasivos y tener buena adherencia ${ }^{6}$.

Actualmente, el método de pesquisa más utilizado por su carácter no invasivo y de bajo costo, es el test de sangre oculta en deposiciones (TSOD) con el cual se ha logrado reducir la mortalidad por CCR en el grupo sometido a pesquisa ${ }^{7-10}$. En los últimos años se incorporó el TSOD inmunológico (TSODi) que ha permitido mejorar la adherencia de la población bajo pesquisa. Otras opciones como la pesquisa con colonoscopia no se utilizan a nivel masivo por su mayor costo, invasividad y requerimientos de personal entrenado ${ }^{11,12}$.

En Estados Unidos de Norteamérica el aumento del diagnóstico precoz producto de la pesquisa podría ser una de las causas que expliquen la reducción de la mortalidad por CCR de 25 a 17 por 100.000 habitantes entre 1990 y 2005. En caso de mantenerse esta tendencia, se proyecta una disminución de la mortalidad a 13/100.000 habitantes en el año $2020^{13}$.

En Chile no se dispone de programas establecidos de pesquisa a nivel poblacional. El objetivo de este estudio es analizar los resultados de un programa de pesquisa de CCR basado en el TSODi en población mayor de 50 años.

\section{Materiales y Métodos}

El año 2007 se diseñó un programa prospectivo multicéntrico, de pesquisa de CCR en población asintomática de 50 o más años y basado en el TSODi OC sensor micro ${ }^{\circledR}$. Para esto se identificaron centros regionales en los cuales hubiesen equipos constituidos para la resolución endoscópica y quirúrgica del CCR y además que estuviesen dispuesto a participar en un protocolo y asistir a un curso de capacitación. En este curso se capacitó a una enfermera por cada centro para que realicen la educación de la población, coordinen el programa e incorporen la información en una base de datos. Al médico coordinador de cada equipo se le instruyó en el modelo de evaluación de los pacientes, selección de pacientes para endoscopia y conceptos terapéuticos. De este modo se constituyó un equipo de trabajo con médicos de las regiones II, IV, VIII, XII, XIV y Metropolitana. Esto se desarrolló mediante tres campañas anuales entre los años 2007 y 2009. En las que se incluyó una charla educativa realizada por una enfermera coordinadora, evaluación por médico, TSODi, colonoscopia y biopsia según hallazgos (Figura 1). En cada campaña, los pacientes pagaron un valor único por la atención médica y el TSODi (precio preferencial) y la colonoscopia fue realizada con copago cero (sólo cobertura del seguro).

Durante la evaluación médica se identificaron los antecedente familiares y síntomas sospechosos (hematoquezia, cambio de hábito intestinal y dolor abdominal prolongado) siendo enviados directamente a colonoscopia aquellas personas que presentaran estos antecedentes o síntomas.

En caso de no poseer factores de riesgo para CCR se le entregó el test inmunológico de sangre oculta en deposiciones (TSODi) (OC-sensor Micro, Eiken Chemicals Co. Ltd, Tokio, Japón), el cual es específico y cuantitativo por aglutinación de látex con anticuerpos monoclonales anti hemoglobina humana. El TSODi no requiere restricciones nutricionales especiales ni suspensión de medicamentos. De cada individuo estudiado se analizaron dos muestras independientes de deposiciones (tomadas en días sucesivos), y como límite de positividad se definió una concentración de hemoglobina igual o mayor a $100 \mathrm{ng} / \mathrm{mL}$ en cualquiera de las muestras.

El material entregado a cada paciente constaba de 2 tubos para extraer muestras de dos deposiciones diferentes junto a un instructivo para la toma de muestra. Los tubos, posterior al muestreo fueron analizados en forma centralizada en el Laboratorio de Oncología y Genética Molecular de Clínica Las Condes. Los resultados del TSODi fueron enviados a las respectivas enfermeras coordinadoras quienes citaron a aquellos individuos con resultados positivos a colonoscopia completa en su centro de base.

A los pacientes con resultado negativo se les indicó control anual de acuerdo a las pautas de seguimiento actual.

Todos los pacientes con TSODi positivos fueron citados para un estudio endoscópico. En las colonoscopias en las cuales se detectaron y resecaron pólipos estos fueron enviados a estudio 


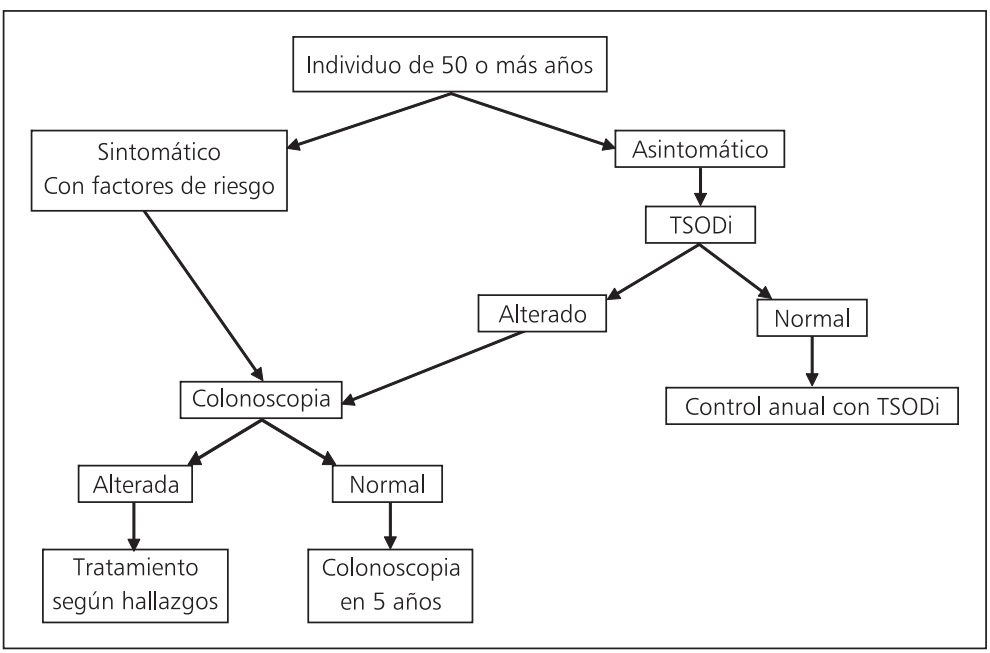

Figura 1. Diagrama de estudio y manejo de pacientes. centralizado por un mismo anatomopatólogo. En ellos se estudió la clasificación histológica (hiperplástico, serrado, tubular, tubulovelloso) y la displasia en bajo y alto grado.

Los adenomas de alto riesgo fueron definidos como aquellos mayores de $1 \mathrm{~cm}$, presencia de más de 3 pólipos, componente velloso y/o el hallazgo de displasia severa.

El criterio de cáncer invasor se utilizó al observar células neoplásicas más allá de la muscular de la mucosa. La información obtenida tanto de los resultados de TSODi, estudio endoscópico, anatomopatológico y de cirugías fue registrada en forma prospectiva por la enfermera a cargo en cada centro en una base de datos centralizada.

\section{Resultados}

En el período descrito se evaluó un total de 6.348 pacientes (Tabla 1 ). En el primer, segundo y tercer año se evaluaron 3.492, 2.096 y 760 pacientes, respectivamente.

Se realizaron un total de 4.938 TSODi (77\% del total de evaluados), de ellos 476 (9,6\%) fueron positivos para sangre oculta.

Se indicó un total de 2.359 colonoscopias, siendo 476 de ellas debido a un OC sensor positivo y 1.883 por presentar síntomas o tener factores de riesgo. Del total de endoscopias solicitadas, se realizaron 1.184 lo cual evidencia una adherencia global de 50,1\% (Tabla 2). No se obtiene información detallada de las causas por las cuales los pacientes no se realizaron las colonoscopias indicadas, pero la mayoría mencionó temor al examen o dificultad de coordinarla con su actividad laboral. Se estima que el factor económico fue el menos relevante dado el diseño de copago cero.

Analizado por subgrupos se observó que de los pacientes con TSODi con resultado positivo, se realizaron 279 colonoscopias, lo que representa una adherencia en este grupo de 58,6\% comparado con las 1.883 colonoscopias indicadas por presencia de factores de riesgo de las cuales se realizaron 905 , lo que implica una adherencia de $48,1 \%$.

Tabla 1. Descripción de evaluaciones de pacientes y colonoscopias realizadas según centro de salud

\begin{tabular}{|lcc|}
\hline Evaluaciones & $\begin{array}{c}\text { Colonoscopias } \\
\text { realizadas }\end{array}$ \\
\hline Centro 1 & 517 & 25 \\
\hline Centro 2 & 723 & 11 \\
\hline Centro 3 & 823 & 129 \\
\hline Centro 4 & 591 & 39 \\
\hline Centro 5 & 414 & 24 \\
\hline Centro 6 & 424 & 15 \\
\hline Centro 7 & 2.096 & 573 \\
\hline Centro 8 & 760 & 368 \\
\hline Total & 6.348 & 1.184 \\
\hline
\end{tabular}


Tabla 2. Análisis de resultados del OC-sensor y estudio colonoscópico según año del estudio

\begin{tabular}{|cccccc|}
\hline Año & OC indicados & OC realizados & OC (+) & OC (+) con colonoscopia & OC (+) con pólipos \\
\hline 2007 & 3.492 & $3.172(90,8 \%)$ & $295(9,3 \%)$ & 170 & 72 \\
2008 & 2.096 & $1.076(52 \%)$ & $112(10,4 \%)$ & 65 & 22 \\
\hline 2009 & 760 & $690(90,7 \%)$ & $69(10 \%)$ & 44 & 18 \\
\hline Total & 6.348 & 4.938 & $476(9,6 \%)$ & $279(58,6 \%)$ & $112(40,1 \%)$ \\
\hline
\end{tabular}

Tabla 3. Detección de pólipos según año del estudio

\begin{tabular}{|cccc|}
\hline Año & $\begin{array}{c}\text { Total } \\
\text { pólipos }\end{array}$ & $\begin{array}{c}\text { Pólipos de } \\
\text { alto riesgo }\end{array}$ & $\begin{array}{c}\text { Cáncer } \\
\text { colo-rectal }\end{array}$ \\
\hline 2007 & 127 & 28 & 11 \\
\hline 2008 & 221 & 36 & 2 \\
\hline 2009 & 128 & 11 & 0 \\
\hline Total & 476 & 75 & 13 \\
\hline
\end{tabular}

El porcentaje de llegada al ciego en el total de colonoscopias fue de $88 \%$. Esta cifra es variable dependiendo del año, ya que el primer año, en el cual se incluyó una mayor cantidad de centros, el porcentaje de llegada al ciego fue de $58 \%$, comparado con $97 \%$ del segundo y tercer año, cuando el programa fue realizado en un solo centro.

El TSODi fue positivo en 476 pacientes $(9,6 \%)$ y se realizaron la colonoscopia 279 de ellos $(58,6 \%)$.

En un total de 1.184 colonoscopias realizadas, se encontraron 476 pacientes portadores de pólipos $(40,2 \%)$ siendo adenomatosos 304 de ellos $(63,8 \%)$ y de alto riesgo 75 pacientes $(16 \%)$ (Tabla 3).

En trece pacientes se hizo el diagnóstico de adenocarcinoma, es decir, el 1,1\% de quienes fueron sometidos a una colonoscopia. En tres de ellos el adenocarcinoma se encontraba dentro de un adenoma y al cumplirse los criterios de seguridad de la polipectomía endoscópica, no fue necesario continuar con una cirugía. En los 10 pacientes operados los tumores fueron diagnosticados en etapas tempranas (etapa I y II) sin metástasis linfonodales ni a distancia.

Por cada mil personas evaluadas en el programa se encontraron 75 portadores de pólipos, 12 con pólipos de alto riesgo y 2 con adenocarcinoma.

\section{Discusión}

En Chile, el CCR es una amenza ya que se encuentra dentro de las primeras cinco causas de muerte por cáncer en Chile y en los últimos 20 años la mortalidad derivada de este tumor se ha duplicado ${ }^{1}$. El hecho de no contar con indicadores de incidencia a nivel nacional -dado que Chile no cuenta con un Registro Nacional de Tumores- no nos permite una aproximación más precisa de la magnitud de esta enfermedad. De acuerdo a nuestra proyección económica y siguiendo la curva de mortalidad, es esperable que la tendencia de del CCR en Chile siga en aumento y se alcance pronto la incidencia de los países desarrollados. Sin embargo, hay lugares en Chile donde ya se superó la mortalidad de Estados Unidos de Norteamérica como es el caso de Punta Arenas cuya tasa es de 19,6/100.000. El CCR presenta algunas características específicas para ser considerado en programas de pesquisa como es el hecho de contar con un marcador que es el pólipo y una evolución conocida (secuencia adenoma-carcinoma). Este el primer estudio multicéntrico que trata de ejecutar un programa de pesquisa a nivel nacional. Este estudio, se realizó en individuos mayores de 50 años, grupo de edad que agrupa a 4.121.229 personas en Chile, según la estimación del Instituto Nacional de Estadística al año 2010.

Respecto al examen de pesquisa utilizado en este estudio (OC sensor micro), debemos destacar que los únicos programas de pesquisa que han logrado reducir la mortalidad por CCR se han basado en el TSOD ${ }^{7,8}$. En el presente trabajo se utilizó un TSODi, el cual no necesita de restricciones alimentarias ni medicamentosas ${ }^{10,14}$. Además, su análisis es automatizado, lo cual disminuye los errores de interpretación ${ }^{15}$.

En este estudio se alcanzó una adherencia general de 77\% al OC-sensor, cifra que bordea el 
Programa de detección de neoplasias colorrectales - F. López-Köstner et al

límite superior de lo descrito en la literatura ${ }^{10,16}$ lo que se podría atribuir al hecho de haber utilizado un test inmunológico ${ }^{17,18}$. La positividad del OC sensor en este programa se enmarca en los porcentajes descritos internacionalmente, los que oscilan entre 5 y $12 \%{ }^{10,16,17}$.

Respecto a la indicación de estudio endoscópico, este fue solicitado tanto a pacientes asintomáticos con positividad del TSODi, así como a pacientes con factores de riesgo para presentar CCR. Este último grupo fue el más numeroso, lo cual puede explicarse porque al ser esta la primera campaña masiva, las personas acudieron con el fin de solucionar síntomas que poseían y no solamente detectar problemas en pacientes asintomáticos.

La adherencia para efectuarse los estudios endoscópicos fue superior en los pacientes a quienes se les indicó por TSODi positivo, que en los sintomáticos. Esto es esperable, dado que la campaña incluía diversas charlas en las cuales se remarcaba la necesidad de realizarse un estudio endoscópico luego de un resultado positivo.

Ya que en este tipo de intervenciones se logra identificar a pacientes asintomáticos, en teoría se lograría identificar a pacientes con mayor probabilidad de tener lesiones preneoplásicas o CCR en estadío temprano. Esto se confirma en este trabajo, ya que en los 13 casos de pacientes con CCR, la estadificación mostró estadios no metastásicos.

En el ámbito internacional, en programas realizados en Estados Unidos de Norteamérica, $40 \%$ de los pacientes se logra diagnosticar en etapas localizadas, con una sobrevida de $90 \%$ a 5 años en estos $\operatorname{casos}^{19}$. En Europa en el año 2003 se inició una política de pesquisa de CCR, la que ha sido implementada completa o parcialmente en la mayoría de los países de la Comunidad Europea. Un reporte inglés y otro escocés demostraron que luego de iniciar programas de pesquisa se logra detectar a pacientes con CCR es estadíos más tempranos al compararlos con lo detectado tradicionalmente ${ }^{20,21}$. Resultados semejantes se obtienen en España al implementar un programa en pacientes entre 50 y 69 años ${ }^{22}$.

En Australia, uno de los países con más alta incidencia de CCR en el mundo, durante los años 2002 y 2003 se implementó un programa piloto a nivel regional de pesquisa de CCR en base a $\mathrm{TSOD}^{23}$. Los resultados satisfactorios en la primera etapa impulsaron al desarrollo de un plan a nivel nacional, el cual se desarrolla desde el 2006. Este programa incluyó el estudio de individuos entre 55 y 65 años. El informe preliminar del año 2009, muestra una diferencia significativa en la etapa del diagnóstico de CCR al comparar la población asintomática diagnosticada por pesquisa $(40 \%$ en etapa I y $3 \%$ en etapa IV) y la población sintomática ( $14 \%$ de casos en etapa I y $15 \%$ en etapa IV $)^{4}$. Otro aporte de un programa de pesquisa es detectar las lesiones neoplásicas de alto riesgo, que en este trabajo corresponden a 75 pacientes. A ellos se les resecó la lesión con lo cual se evitó la progresión de las lesiones al cáncer. Lo anterior es uno de los principales factores al analizar en los programas de prevención ${ }^{24}$.

Respecto a la calidad de los estudios endoscópicos realizados destaca que en todos los casos se indicaron colonoscopias completas. Este procedimiento permite medir parámetros correlacionados con estándares de calidad, como la llegada al ciego. En este estudio, la llegada al ciego fue de $88 \%$, lo cual se enmarca dentro del límite inferior de lo aceptado internacionalmente. Sin embargo, esta cifra varía en los 3 años, siendo menor el primer año con respecto a los dos siguientes. Los motivos para tal diferencia pudiesen explicarse por la mayor diversidad de centros que realizaron las colonoscopias en el primer año, ya que el segundo y tercer año las endoscopias fueron realizadas por un grupo más reducido de médicos de una misma institución. Algunos de los centros realizaban un bajo número de colonoscopias, lo que podría inducir a un error estadístico tipo 2. Al ser esta la primera experiencia multicéntrica de pesquisa en CCR, queda claro la importancia de estandarizar el exámen endoscópico y certificar a centros que cumplan con los objetivos para trabajar en pesquisa (estandar de preparación intestinal, sedación del paciente, llegada al ciego, tiempo de retiro del instrumento y eficiencia del flujo de los pacientes por parte de todo el personal del centro endoscópico.

Otra de las limitaciones de este programa es la falta de información del seguimiento de los pacientes quienes no se realizaron una colonoscopia con un TSODi positivo o de la adherencia futura para continuar con el TSOD anual o el seguimiento endoscópico en aquellos con extirpación de pólipos. Creemos que es fundamental contar con una enfermera de seguimiento que permita la coordinación y vigilancia de la población en riesgo.

En resumen, este trabajo constituye la primera experiencia multicéntrica a nivel nacional de pes- 
quisa de CCR. El trabajo demuestra que nuestra población presenta una alta adherencia a la realización del TSODi y fue posible la detección precoz y tratamiento de un alto porcentaje de lesiones neoplásicas de alto riesgo.

\section{Referencias}

1. Disponible en DEIS. Dirección: http://deis.minsal.cl/ index.asp

2. Jemal A, Siegel R, Ward E, Hao Y, Xu J, Thun MJ. Cancer statistics, 2009. CA Cancer J Clin 2009; 59: 225-49.

3. Frazier AL, Colditz GA, Fuchs CS, Kuntz KM. Costeffectiveness of screening for colorectal cancer in the general population. JAMA 2000; 284: 1954-61.

4. Ananda SS, McLaughlin SJ, Chen F, Hayes IP, Hunter AA, Skinner IJ, et al. Initial impact of Australia's National Bowel Cancer Screening Program. Med J Aust 2009; 191: 378-81.

5. Vogelstein B, Fearon ER, Hamilton SR, Kern SE, Preisinger AC, Leppert $\mathrm{M}$, et al. Genetic alterations during colorectal-tumor development. N Engl J Med 1988; 319: 525-32.

6. Bond JH. Fecal occult blood tests in occult gastrointestinal bleeding. Semin Gastrointest Dis 1999; 10: 48-52.

7. Kronborg O, Fenger C, Olsen J, Jørgensen OD, Søndergaard O. Randomised study of screening for colorectal cancer with faecal-occult-blood test. Lancet 1996; 348 : 1467-71.

8. Hardcastle JD, Chamberlain JO, Robinson MH, Moss SM, Amar SS, Balfour TW, et al. Randomised controlled trial of faecal-occult-blood screening for colorectal cancer. Lancet 1996; 348: 1472-7.

9. Mandel JS, Church TR, Bond JH, Ederer F, Geisser MS, Mongin SJ, et al. The effect of fecal occult-blood screening on the incidence of colorectal cancer. $\mathrm{N}$ Engl J Med 2000; 343: 1603-7.

10. Allison JE, Sakoda LC, Levin TR, Tucker JP, Tekawa IS, Cuff $\mathrm{T}$, et al. Screening for colorectal neoplasms with new fecal occult blood tests: update on performance characteristics. J Natl Cancer Inst 2007; 99: 1462-70.

11. Levin TR. Flexible sigmoidoscopy for colorectal cancer screening: valid approach or short-sighted? Gastroenterol Clin North Am 2002; 31: 1015-29.

12. Mergener K. The role of CT colonography in a colorectal cancer screening program. Gastrointest Endosc Clin N Am 2010; 20: 367-77.

13. Haggar FA, Boushey RP. Colorectal cancer epidemiolo- gy: incidence, mortality, survival, and risk factors. Clin Colon Rectal Surg 2009; 22: 191-7.

14. Greenwald B. From guaiac to immune fecal occult blood tests: the emergence of technology in colorectal cancer screening. Gastroenterol Nurs 2005; 28: 90-6.

15. Vilkin A, Rozen P, Levi Z, Waked A, Maoz E, Birkenfeld $S$, et al. Performance characteristics and evaluation of an automated-developed and quantitative, immunochemical, fecal occult blood screening test. Am J Gastroenterol 2005; 100: 2519-25.

16. Fenocchi E, Martínez L, Tolve J, Montano D, Rondán M, Parra-Blanco A, et al. Screening for colorectal cancer in Uruguay with an immunochemical faecal occult blood test. Eur J Cancer Prev 2006; 15: 384-90.

17. Van Rossum LG, van Rijn AF, Laheij RJ, van Oijen MG, Fockens P, van Krieken HH, et al. Random comparison of guaiac and immunochemical fecal occult blood tests for colorectal cancer in a screening population. Gastroenterology 2008; 135: 82-90.

18. Guittet L, Bouvier V, Mariotte N, Vallee JP, Arsène D, Boutreux S, et al. Comparison of a guaiac based and an immunochemical faecal occult blood test in screening for colorectal cancer in a general average risk population. Gut 2007; 56: 210-4.

19. Edwards BK, Ward E, Kohler BA, Eheman C, Zauber AG, Anderson RN, et al. Annual report to the nation on the status of cancer, 1975-2006, featuring colorectal cancer trends and impact of interventions (risk factors, screening, and treatment) to reduce future rates. Cancer 2010; 116: 544-73.

20. Ellul P, Fogden E, Simpson CL, Nickerson CL, McKaig BC, Swarbrick ET, et al. Downstaging of colorectal cancer by the National Bowel Cancer Screening programme in England: first round data from the first centre. Colorectal Dis 2010; 12: 420-2.

21. Steele RJ, McClements PL, Libby G, Black R, Morton C, Birrell J, et al. Results from the first three rounds of the Scottish demonstration pilot of FOBT screening for colorectal cancer. Gut 2009; 58: 530-5.

22. Navarro M, Peris M, Binefa G, Vanaclocha M, Losa F, Fernández E. Colorectal cancer in a population with a guaiac-based screening programme. Med Clin (Barc) 2009; 132: 495-500.

23. Millard FB. Bowel cancer screening in Australia. Aust Fam Physician 2006; 35: 188-91.

24. Ginsberg GM, Lim SS, Lauer JA, Johns BP, Sepúlveda CR. Prevention, screening and treatment of colorectal cancer: a global and regional generalized cost effectiveness analysis. Cost Eff Resour Alloc 2010; 8: 2. 